\title{
CONFLICTS OF INTEREST AT ANY GOVERNANCE LEVEL OF EU FUND MANAGEMENT
}

\author{
LELA KAMLADZE
}

https://doi.org/10.35945/gb.2017.03.012

Doctor of Economics,

Visiting Specialist at Akaki Tsereteli Kutaisi State University, Georgia

KEYWORDS: CONFLICT OF INTEREST, GOVERNANCE LEVEL, MANAGEMENT OF EU FUNDS

This study aims to understand how the European Union member state administrations is involved in the shared management of EU funding (e.g. the European Structural and Investment (ESI) Funds), ensure the integrity of relevant decision-making and management processes. The study takes an interest in both, 'soft' and 'hard' law approaches, e.g. codes of conduct and legislation on the prevention of Conflicts of Interest. The Specifications for the study rightly emphasise 'the high levels of administration at the regional level' (p. 2). Indeed, it is at this level, that in many member states most of the relevant decisions regarding the deployment of the European Structural and Investment (ESI) Funds are taken, and that therefore require attention to avoiding Conflicts of Interest. Finally, the study aims not only to establish an overview of the existing approaches (comparing, inter alia, between legislation and codes of conduct) to addressing Conflicts of Interest, but also takes an interest in their effectiveness, and in possible future improvements, drawing, inter alia, on best practices in and outside the EU.

Several research tools were deployed to answer the research questions:

1. Desk research;

2. Case studies.

Until today, the competence for implementing Community law has, as a principle, stayed with the MS, and their organisational and institutional structures are protected under the procedural autonomy doctrine. Moreover, the principles of subsidiarity, proportionality, enumerated competence and safeguarding national identity and institutional autonomy of the MS reflect this classic picture of clear delineation between the $\mathrm{EU}$ and the national administrations.

As a consequence of the distribution of tasks and the functional separation between the EU and the member sat as a clear area of tension exists between the principle of institutional autonomy of the MS and the duty to 'implement' community policies effectively, as enshrined in Article 197 paragraph 1 TFEU. During the past decades the European Court of Justice has interpreted concepts such as the 'correct implementation of community policies' more strictly and linked it to the principle of effectiveness. This also applies to the duty to effectively safeguard community financial interests.

Despite the fact that (as a principle) implementation falls under MS responsibility, it is certainly true that especially as regards the implementation of the ESI Funds, today, there is no more a clear dividing line between EU administrative law and national administrative law and also not between EU administration and national administration as the scholarly discussions on the Europeanisation of public law, and Europeanisation of administrative law illustrate. For example, forms of administrative 'engrenage' can best be observed as regards the effects of the so-called partnership principle which links European, national, regional and local administrations. Another example for the 'fusion' of administrations is OLAF which assumes important monitoring and surveillance functions as regards the protection of community financial interests on the European but also on the national and regional level. Notwithstanding, OLAF and the EC rely on the 'goodwill' of the MS to implement the EU funds effectively.

The EC has only few possibilities to monitor and to enforce its own policies on the local level. Therefore, the EC is using other channels to make sure that (not only) community financial interests are properly implemented. For example, the EC is funding various public administration reforms in many EU Member states and uses the ESF to support capacity building, anti-fraud- and anti-corruption policies etc.. Implementing European Col requirements in the field of the ESI Funds faces additional challenges compared to the national level. In its report 'Making the best use of EU money: a landscape review to the risks of the financial management of the EU budget', 30 the European Court of Auditors discuss a large variety of reasons and factors which explain risks in the implementation of EU funds. Corruption, fraud and conflict of interests are one reason, amongst many others. One reason can also be found in the legal and institutional structure of the EU and the lack of powers as regards the implementation of EU policies on the national level, weak monitoring and enforcement capacities and the existence of an accountability gap on the side of the MS: 'Member States are obliged to report only on the elements that are included in the legal basis on monitoring and reporting. If the above cases are not considered irregularity they will not be mentioned in the irregularity reports either. Nevertheless, as the EC participates in the MC meetings it can pay attention on the extent to which the guidance is applied and discuss this with the MAs in bilateral review meetings' (DG EMPL).

Another problem concerns the above mentioned 'accountability gap'. While the MS are responsible for spending approximately $80 \%$ of the EU budget under shared management arrangements, it is the EC that is ultimately responsible for supervising and implementing the EU budget, in turn, overseen by the EP's Committee on Budgetary Control, e.g. in the context of the discharge procedure. As DG Employment replied to this study: 'EC communicates related 
rules to the ESIF Managing Authorities, which could be both at national or regional level.

The formal channel is the expert group (the ESF technical working group, for example) where all guidance is presented and discussed. From time to time, there are meetings of that group specifically dedicated on fraud prevention, where OLAF also takes part and presents their experience and recommendations. Due to the big number of managing authorities however not all of them are represented in the expert groups. Therefore, in some countries with several dozens of OP the only channel is the direct communication between the geographical desks, managing the OPs and the respective national/regional bodies. The role of the national coordination and audit bodies in these cases also is critical'.

According to Mögele (2016), this form of institutional cooperation produces a paradox: Although MS are actively involved in the management and implementation of the $\mathrm{EU}$ budget and accountable to the EC (and may be sanctioned in case of unprofessional spending), it is the EC which takes ultimate responsibility. This is also confirmed by the Art. 59 of the EU Budget which gives the political responsibility for the execution of the budget to the EC. Accordingly, the EC is also accountable for those acts for which the MS are responsible. This again means that - on the national level the monitoring of EU funds is not taken care of in the same way as the national funds and accountability mechanisms differ as regards the implementation of EU funds.31 Since EU funds are spent via 28 national administrations and many regional and local authorities with unequal administrative capacity (skills and resources) this increases the risk of errors occurring, as well as the risk of poor quality spending.

As our case studies have shown, the biggest challenge is not the lack of rules and codes, but the lack of awareness about potential Col, the lack of ethical leadership in MCs, the lack of transparency, and the poor management and the institutionalisation of Col policies in the MCs. In most cases, more regulation is not required in those situations or countries where high levels of public trust exist. We believe that the existing rule-based approaches are necessary, but must be complemented by soft approaches, ethical leadership and investments in transparency. A transparent system that can be observed by everyone as a matter of course will also demonstrate to members of the public and others who deal with the implementation of the ESI Funds that the MCs perform their role in a way that is fair and unaffected by improper considerations. Therefore, we believe that protocols and voting behavior should be published and Col more intensively discussed. The latter requires an active role by the MC chairperson.

We are also critical as regards the effectiveness of codes and guidelines. Adopting a code of conduct is not sufficient. In most MCs, EU-guidelines are not well known. Also on the national level, much time and energy is usually spent in designing, formulating, and adopting a code but many institutions stop here. The code remains a 'paper tiger' and is never implemented or monitored. The future challenge should be to 'utilise the dynamics' which have emerged from the formulation of the code. This will support a continuous process of reflection on the central values and standards contained in the code. Thus, it would be important, if politicians and public servants meet on a regular basis and discuss (and update) the existing code (s).

Overall, Ethics and Col policies should not be a 'plugin policy' that fills the gaps that other policies and other governance logics produce. It is time to acknowledge that ethics is not only a normative question. It is a practical, daily-life issue. Most important thing is to have a credible monitoring and control mechanism in place, the crucial issues being transparency and accessibility of information, monitoring and enforcement. While we do not suggest the introduction of more rules and more codes, we believe that countries should invest in monitoring (the effectiveness of their own) Col policies and nominate existing bodies (e.g. the ombudsman) to carry out regular Col tests and reports.

Col policies are an expression of distrust. We believe that in the future, the public will continue to question practices where public institutions and/or politicians regulate their own ethical conduct. Any form of self-regulation will continue to cause suspicion. This also relates to the MCs. Our findings suggests that it may be advisable to establish an independent Col commission and/or independent compliance officer who should carry out these tasks.

Size of a country and relationship with Col - the case of Estonia. With a population of under 1.3 million, Estonia is one of the smallest countries in Europe. The size of the population influences $\mathrm{Col}$ in the public sector. However, this does not suggest that only because Estonia is a small country, it has higher levels of Col (in reality, it has relatively low levels of corruption, fraud and Col). Still, the size of the country relates to Col. First, because few actors are working in the administration. This again means that people know each other (personally) which again means that decision making, coordination and communication structures are less formalised and anonymous than in bigger administrations. Thus, the fact that the public workforce is small may have positive (side-effects through more possibilities for social control of actors) and/or negative effects because of enhanced possibilities to create networks and personal ties which make it more difficult to maintain strictly formalised decision-making procedures and processes. Another problem is the high labour turnover in the public sector. High turnover favours high levels of interaction between the public and private sectors, facilitates corruption and weakens institutionalised knowledge. This again may support the appearances of Col.

Overall, in all of our case-studies there is no evidence pointing to much awareness of Col as affecting the MCs or related sub-committees. In many cases, stakeholders consider that the issue of $\mathrm{Col}$ is not relevant in the first place in the context of the MCs. This is somewhat contradictory since the $\mathrm{MC}$ rules of procedure, in some (but not all) cases actually refer to Col, thus addressing the requirements spelled out in the EU regulatory framework for the ESI Funds. However, there is no specific guidance on Col in the context of the MCs. As regards the implementation of $\mathrm{Col}$ in the $\mathrm{MC}$ meetings, we conclude that, in most cases, there is no operationalisation of 
the Col rules (where they exist). The lack of operationalisation is explained by a lack of awareness that Col can in fact become relevant for MCs. Moreover, as the ES case illustrates, the focus is strongly on anti-fraud measures, looking primarily at public procurement.

For many years, international research on ethics and integrity has focused on the characteristics and prevalence of high performance ethics infrastructures. Much of this literature assumes that high performance ethics infrastructures constitute 'best practice' and universally applicable management, although a distinction can be drawn between those arguing for a contextual best-fit approach and those arguing for more of a best-practice approach, based on a belief in the more universal advantages of these systems. The best-practice approach is based on the belief that ethics infrastructures can be used in any organisation and the view that all organisations can improve performance if they identify and implement best practices.

In the meantime, there is considerable consensus on what constitutes bad practices, for example, the absence of codes of ethics, poor leadership, unfair HR policies, lack of training, unprofessional performance measurement etc..

However, it is much more difficult to identity institutional best practices, although the search for benchmarks is becoming ever more popular. Still, it is possible to continue the work on 'common elements' and 'good practices' that really work in the field of Col. The contours of an approach to establishing effective Col policies are steadily coming into view, and comprise aspects such as measurement of Col; strengthening the focus on transparency, openness and accountability; supporting efforts to tackle Col through cycles of awareness raising and learning about the risks of Col; systematic monitoring and evaluation of effectiveness; and paying more attention to implementation, compliance and results and not only the implementation of rules.

Overall, the search for best ethics infrastructures is confronted with a context and institutionbased, fragmented and pragmatic reality. Overall, institutional differences notably the levels of budgetary resources, social legitimacy, work systems, labour markets, education and training systems, work organisation and the collective organisation of employers and employees - mediate the impact of converging processes.

Consequently, the proposition for implementing institutional and organisational best practice models such as ethics infrastructures is ambiguous. In fact, the political and institutional world is currently moving away from universal or even European best-practice institutional configurations towards more specific best-fit context-related models. New developments lean more towards the testing of new innovative organisational models and work systems that fit into the national, regional, local or even organisational context.
Current discussions in the field of Col also turn away from the 'grand old' dichotomy: valuebased approaches versus compliance-based approaches. This can best be seen in the field of Col, where countries have started to realise that the management of Col does not work without clear rules, formal procedures, and strong enforcement mechanisms but also not without softinstruments, awareness raising, strong leadership, independent ethics committees, registers of interest and more and better management capacity. Overall, countries are also starting to test new instruments like staffassessments on Col and integrity, monitoring integrity policies on the governmental level and introducing better registers that collect data on Col violations.

As regards the MCs under the ESI Funds, we have concluded that:

a) There is too little awareness about the importance of Col in MCs; there is also too little discussion on Col. Therefore, we suggest to invest in awareness raising instruments, also at the EU level, through ESF-funded programmes in the fields of Public Sector Modernisation and Capacity Building.

b) Mostly, the MC rules of procedures address Col, if at all, very cursorily. We suggest that each MC should have Col guidelines at hands. There is no need to produce new ones, as best-practices exist (EU guidelines, OECD-toolkits). This is only a matter of better internal distribution and communication.

c) Frequently, MCs are not transparent as regards the management of Col. We suggest to publish protocols of meetings and voting behavior and, for the purpose of research and scrutiny, to collect these reports in publicly available databases (respecting, of course, existing rules on data protection and privacy).

d) Often, the chairperson does not discuss Col in the $\mathrm{MC}$ because priorities are set elsewhere. This is also due to a lack of ethical leadership and awareness as regards the importance of the issue. Here, ethical leadership is important. Chairpersons need to be trained in the field of Col and should be obliged to discuss Col during committee proceedings.

e) Involvement of external experts in the proceedings as well as in training and awareness raising can help eliminate blind spots and identify systemic weaknesses. Experts could, e. g., be consulted in elaborating risk management strategies, providing guidance and instructions as well as distributing roles and responsibilities to identify and mitigate Col risks.

f) Most countries have no information and data on the development of Col under the ESI Funds. There is also very little monitoring in the field of Col. We believe that monitoring and reporting on Col is of utmost importance. Without asking for the establishment of a new ethics/Col bureaucracy, we suggest to ask existing authorities and bodies in the field to regularly report on the development of Col. Good practices exist in NL and NO. 


\section{REFERENCES:}

1. Anechiarico, F. \& Jacobs, J. (1996) The Pursuit of Absolute Integrity. How Corruption Control Makes Government Ineffective.

2. Bertók, J. \& Maesschalck, J. (2009) Public Sector Ethics: an infrastructure. In: OECD (Eds.): No longer business as usual Fighting Bribery and Corruption.

3. Demmke, C. et al. (2008) Regulating Conflicts of Interest for Holders of Public Office in the European Union, European Institute of Public Administration.

4. Lascoumes, P. (2014) Condemning corruption and tolerating conflicts of interest: French arrangements regarding breaches of integrity, in: Auby, J.B., Breen, E. \& Perroud, T. (eds) Corruption and Conflicts of Interest, A Comparative Law Approach. 


\section{CONFLICTS OF INTEREST AT ANY GOVERNANCE LEVEL OF EU FUND MANAGEMENT}

LELA KAMLADZE

https://doi.org/10.35945/gb.2017.03.012

Doctor of Economics,

Visiting Specialist at Akaki Tsereteli Kutaisi State University, Georgia

KEYWORDS: CONFLICT OF INTEREST, GOVERNANCE LEVEL, MANAGEMENT OF EU FUNDS

\section{SUMMARY}

This study reviews the Member States' experience with codes of conduct and conflicts of interest affecting the partnership arrangements under the European Structural and Investment Funds. The focus is on conflicts of interest affecting the Monitoring Committees under the European
Regional Development and European Social Fund. The study reviews the rules and other approaches to deal with conflicts of interest, discusses best practices and ends with conclusions and recommendations advocating a complementary rule and value based approach supported by transparency and ethical leadership. 